\title{
Wireless Mesh Communication Using ZigBee Technology for Military Applications
}

\author{
Dineshkumar Jaiswar ${ }^{1}$, Sanjna S. Repal ${ }^{2}$ \\ ${ }^{1}$ P.G. Student, Department of Electronics and Telecommunication Engineering, MCT's RGIT College, Andheri (W), Mumbai-53, \\ Maharashtra, India
}

${ }^{2}$ Assistant Professor, Department of Electronics and Telecommunication Engineering, MCT's RGIT College, Andheri (W), Mumbai-53, Maharashtra, India

\begin{abstract}
Military forces are very essential part of the security system of a country. During, wars and search operations, our soldiers get injured and many of them become lost. Soldiers are the savior of our nation who protects us from enemy attacks, terrorist activities and from many suspicious activities which can harm the civilians and the nation too. Thus, Soldiers health is very important to us. We have implemented a project which has an ability to track the location and monitor health of the soldiers in real time, who become lost and get injured in the warfield. This system will help to save the time, search and rescue operation efforts of army control unit. The location tracking and health monitoring of soldiers by army base station is done by using GPS module and wireless body area sensor networks (WBASNs), such as temperature sensor and heartbeat sensor. The data coming from Sensors and GPS receiver through microcontroller is transmitted wirelessly using ZigBee module. Also, a soldier can get help from army control room and can communicate with other fellow soldier present within the wireless transmission and reception range using Panic Switch in unfavorable conditions. The wireless communication network is created by ZigBee transceivers using ZigBee Mesh Topology.
\end{abstract}

Keywords: ZigBee, GPS, Temperature sensor, Heartbeat sensor, ARM7 microprocessor.

\section{Introduction}

In today's word, the science and technology is growing rapidly with new inventions, innovations and with advance level of their implementations. These immerging advance and enhance technologies are firmly adopted by the defense services to provide high level security and safety to our soldiers. There are many parameters through which defense services can provide security and safety to the soldiers. In this project, we are providing a wireless embedded system by which the Army base stations can monitor on the heartbeat count and body temperature of soldiers using wireless body area sensor networks (WBASNs) such as temperature sensor and heartbeat sensor [1], [2]. Base stations can also know the location of soldiers by tracking them, through Global Positioning System (GPS) and can guide them to any safe area. Also, the soldier can ask for his location from army control unit in case if he/she feels that he/she is lost or to plan any new strategies against enemies. In this project, all the processes are in real time because of the use of LPC2148 microcontroller embedded with ARM7 microprocessor. The sensed data and the tracked location of soldiers will be transmitted wirelessly through ZigBee Mesh Topology using ZigBee Transceivers [1], [3].

During military operations, one of the fundamental challenges is that the soldiers are not able to communicate with control room and sometimes not even with the other fellow soldiers. Once a troop or a soldier become lost during fight in warfield due to some unfavorable environment or adverse fight conditions, then it becomes very difficult to search them and bring back to the army base station. To overcome these situations, every defence organization needs to design and develop some advance, small, portable and robust system to provide safety measures to their soldiers.
There are many problems which are faced by soldiers during wars in battlefield, like:

1) Sometimes soldiers want to know their location when they become lost but they are not able to do so.

2) Sometimes soldiers need some help during panic situations but they are not able to ask for help.

3)Sometimes soldiers are not able to get medical help when they get injured during war.

The system developed by me is consists of three parts, firstone is small $\mathrm{n}$ portable unit for soldier 1 , second-one is also a small $\mathrm{n}$ portable unit for soldier 2 and third-one is for army control unit. The soldier unit 1 consists of a microcontroller embedded with ARM7 (advanced RISC machine) microprocessor, a GPS tracking device, a ZigBee transceiver, a heartbeat sensor, a temperature sensor, etc. The GPS device is use to track the location of the soldiers with the help of satellite communication system. The heartbeat sensor also called pulse rate sensor is use to sense the pulses or heartbeats of human heart, and temperature sensor is used to sense the temperature of human body. All the Sensors and GPS data are transmitted through a ZigBee module to the army control room, using ZigBee Mesh Topology, which is a low power, low data rate transceiver used to transmit and receive the data wirelessly [1], [2], [3], [4].

In this project, our main aim is to construct the communication between soldiers and army control room and even with other fellow soldiers by using this robust, highly efficient and advanced wireless embedded system. This project helps to solve the above mentioned problems as follows: 


\section{International Journal of Science and Research (IJSR) \\ ISSN (Online): 2319-7064}

Index Copernicus Value (2013): 6.14 | Impact Factor (2015): 6.391

1)Using GPS device, control room can provide proper information about the location of soldiers when it is needed.

2)The soldiers can get help in panic situations by communicating with control room, using Panic Switch through wireless ZigBee technology.

3)By this system, it is easy to provide medical assistance to soldiers when they get injured.

\section{System Architecture}

The Architecture of our system is composed of three parts:

\subsection{Soldier's Unit 1}

This unit consists of two types of body area sensor networks such as temperature sensor and heartbeat sensor. These sensors are used to sense the health parameters of soldiers. Temperature sensor will sense the body temperature of soldier and give that sensed data to microcontroller to measure in ${ }^{\circ} \mathrm{F}$. The heartbeat sensor will sense the pulse rate or heartbeats of soldier and give it to the microcontroller to process and count in beats per minute (BPM) [1], [5], [11], [12].

There is a GPS module used to trace the location of soldiers at any moment from anywhere. The GPS receivers are spacebased satellite navigation systems that provide location and time information in all weather conditions from anywhere on or near the earth. The data coming from GPS receiver will pass to microcontroller through IC MAX232, which converts RS-232 voltage level data to TTL/CMOS voltage level data and vice versa. The IC MAX232 is a dual driver/receiver which converts typically $\mathrm{R}_{\mathrm{X}}, \mathrm{T}_{\mathrm{X}}, \mathrm{CTS}$, and RTS signals.

All the data coming from Sensors and GPS receiver are processed by ARM7 microprocessor embedded in LPC2148 microcontroller. A microprocessor is a single integrated circuit (IC), which is a multipurpose programmable device that takes digital data as input, processes it according to the instructions stored in its memory and provide results as output. The ARM (Advanced RISC machine) processors are based on reduced instruction set computing (RISC), which requires significantly fewer transistors than typical complex instruction set computing (CISC) based processors [1], [5], [6], [9]. This makes them cost efficient, energy efficient and less heat emitting device. These reductions are desirable properties for light, portable and battery powered devices such as smart phones, laptops, tablets, notepads and other embedded systems.

A ZigBee transceiver is used to transmit the data, coming from Sensors and GPS receiver through microcontroller, to the army control room wirelessly. A ZigBee is low cost, low power, wireless mesh network standard especially designed and developed for long battery life devices in wireless controlling and monitoring applications. ZigBee devices have low latency which can further reduce the average current [1], [11], [12].
Additionally, an alphanumeric LCD display is used to display the health parameters (i.e. body temperature and heartbeats) and location information of soldier. Also a Buzzer and a Panic Switch are provided. A soldier can press the Panic Switch to get help in panic situation from army control room and from another fellow soldier within the wireless range. The Buzzer of other fellow soldier will sound when Panic Switch is pressed by the soldier in panic condition.

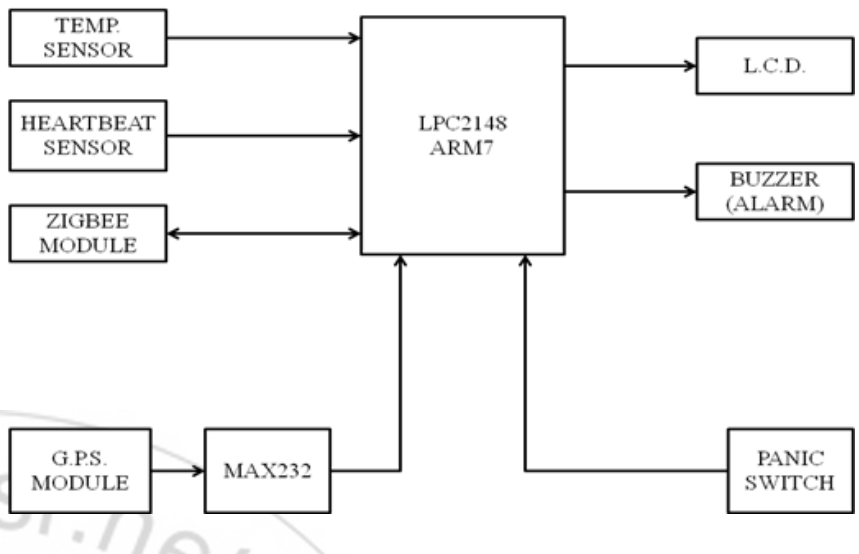

Figure 1: Block Diagram of Soldier's Unit 1

\subsection{Soldier's Unit 2}

This unit mainly consists of an AT89S52 microcontroller which controls all the processes of all unit 2 devices, sensors and modules. It consists of Temperature Sensor to sense the body temperature of the soldier. There is an external 12 bit MCP3204 analog to digital converter in this unit, use to convert the analogous data of temperature sensors into digital data.

This unit also consists of a ZigBee module to transmit and receive data from soldier's unit 1 and control room's unit. It also consists of one LCD to display the alphanumeric data, one Buzzer to generate sound and one Panic Switch which can be used in critical conditions.

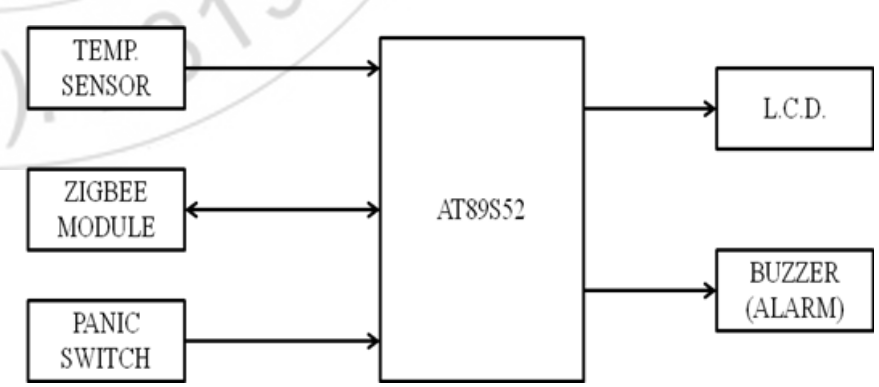

Figure 2: Block Diagram of Soldier's Unit 2

\subsection{Control Room's Unit}

The army control room unit consists of a PC and a ZigBee transceiver. This ZigBee device is interfaced with an USB for easy connection with PC. The ZigBee module will be connected to PC with the help of PL-2303 USB-to-Serial driver installed in that PC [1]. The data coming from ZigBee module will be displayed on PC screen with the help of graphical user interface (GUI) coded using C Sharp language. 


\section{International Journal of Science and Research (IJSR) \\ ISSN (Online): 2319-7064}

Index Copernicus Value (2013): 6.14 $\mid$ Impact Factor (2015): 6.391

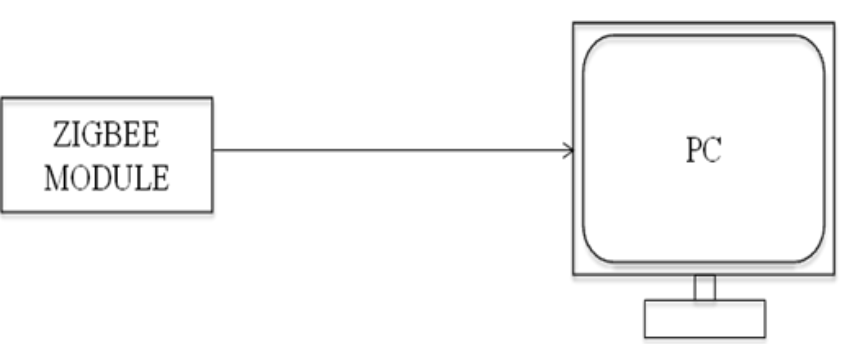

Figure 3: Block Diagram of Control Room's Unit

The wireless communication between all three ZigBee transceivers that we have used in our system, using Mesh Topology is shown in following block diagram.

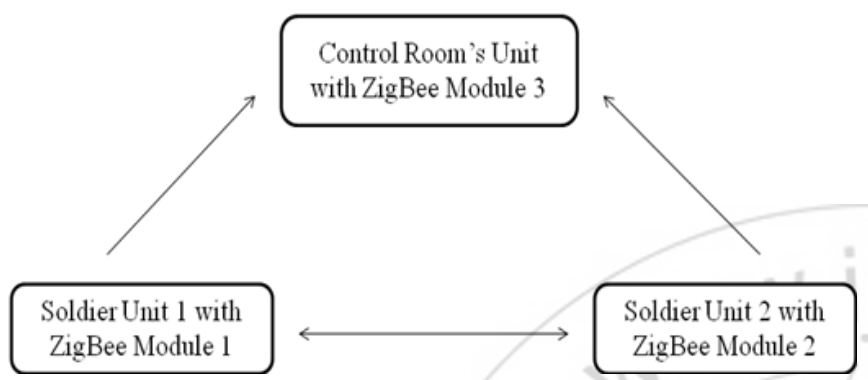

Figure 4: Block Diagram of ZigBee communication using Mesh Topology

\section{Schematics}

The Circuit diagrams or Schematics of our wireless embedded system are presented below:-

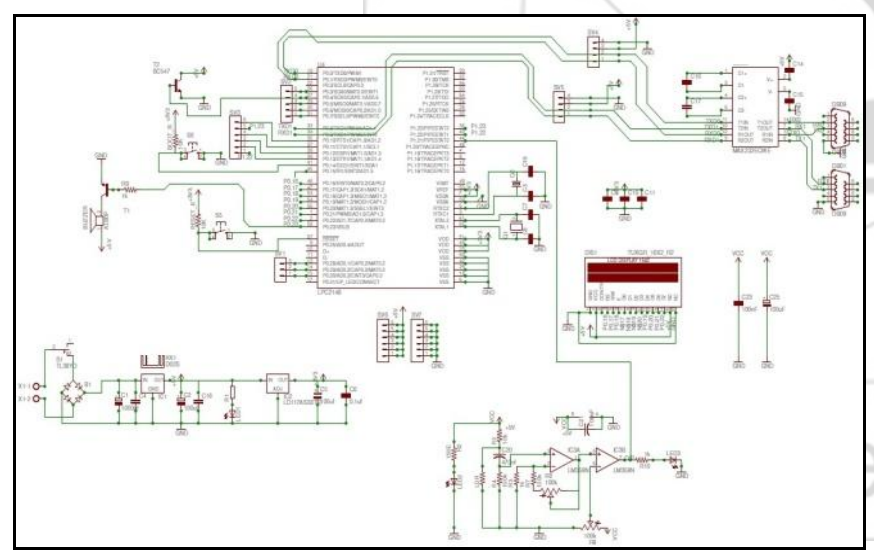

Figure 5: Circuit Diagram of Soldier's Unit 1

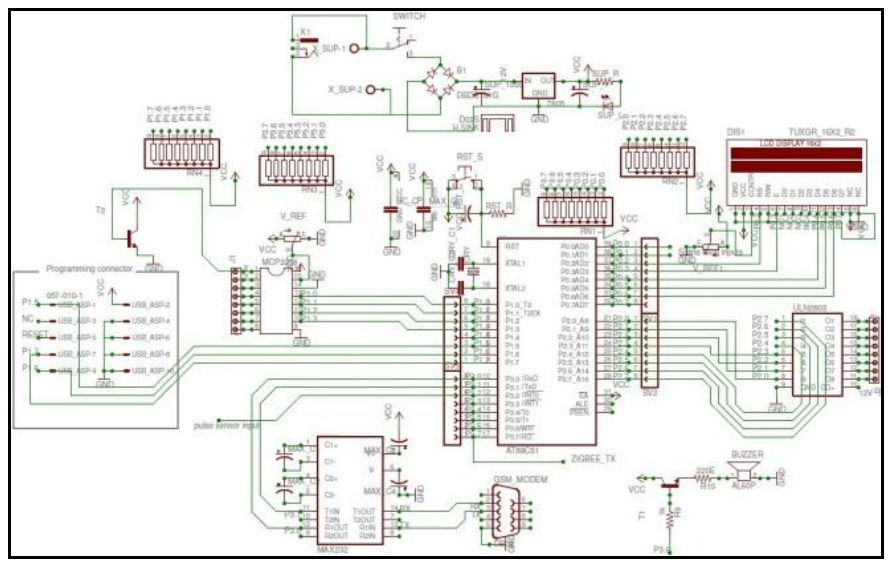

Figure 6: Circuit Diagram of Soldier's Unit 2

\section{Real Hardware Implementation of System}

The images of real hardware designs of our wireless health monitoring and tracking system are presented below:-

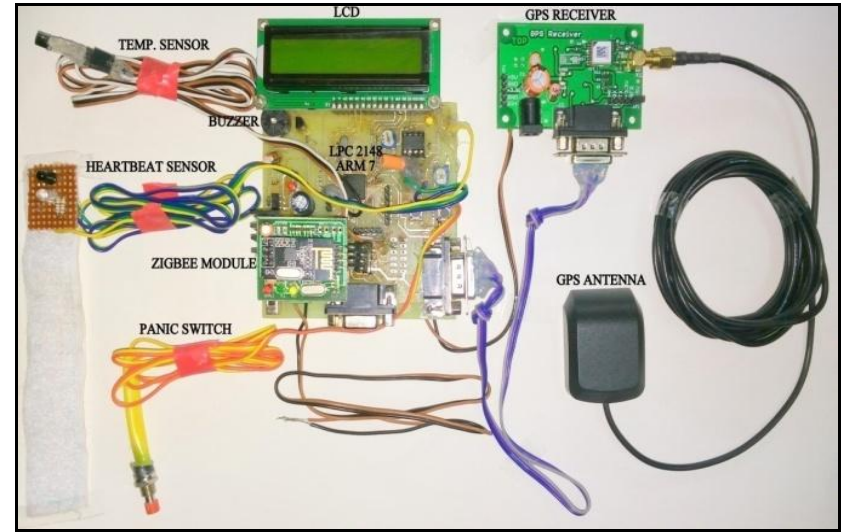

Figure 7: Real Hardware Image of Soldier's Unit 1

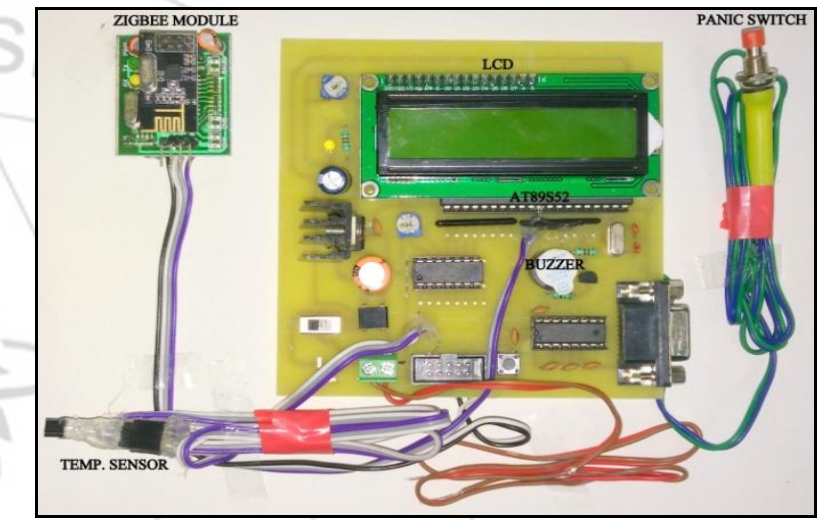

Figure 8: Real Hardware Image of Soldier's Unit 2

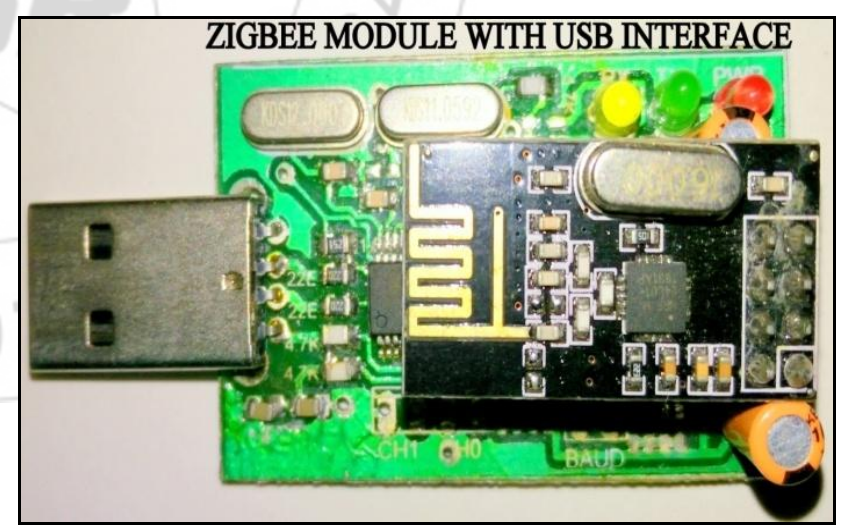

Figure 9: Real Hardware Image of Control Room's Unit

\section{Design Description}

The design description of our system consists of two main sections:

\subsection{Hardware Description}

The microcontroller we are used is the heart of our system. It access and analyze all the data coming from Sensors and GPS receiver. In our project, ARM7 microprocessor, embedded in LPC2148 microcontroller is used to process all the data in real time. To measure body temperature and

Volume 5 Issue 5, May 2016 www.ijsr.net 


\section{International Journal of Science and Research (IJSR) \\ ISSN (Online): 2319-7064}

Index Copernicus Value (2013): 6.14 | Impact Factor (2015): 6.391

heartbeats of soldier, temperature sensor and heartbeat sensor are used. A GPS receiver is used to track the location of soldier. All the data are transmitted and received using wireless ZigBee Mesh Topology through ZigBee Transceivers.

\subsubsection{LPC2148 Microcontroller}

The LPC2148 microcontroller is based on a 32/16 bit ARM7TDMI-S CPU with real-time emulation and embedded trace support, that combines the microcontroller with embedded high speed flash memory of $512 \mathrm{kB}$. A 128-bit wide memory interface and an unique accelerator architecture enable 32-bit code execution at the maximum clock rate up to $60 \mathrm{MHz}$. For critical code size applications, the alternative 16-bit Thumb mode reduces code by more than $30 \%$ with minimal performance penalty. Due to its tiny size and low power consumption, LPC2148 is ideal for applications where miniaturization is a key requirement, such as access control and point-of-sale. A blend of serial communications interfaces ranging from a USB 2.0 Full Speed device, multiple UARTs, SPI, SSP to $\mathrm{I}^{2} \mathrm{C}$-Bus, and on-chip SRAM of $40 \mathrm{kB}$, make this device very well suited for communication gateways and protocol converters, soft modems, voice recognition and low end imaging, providing both large buffer size and high processing power. Various 32-bit timers, dual 10-bit ADCs, single 10-bit DAC, PWM channels and 45 fast GPIO lines with up to nine edge or level sensitive external interrupt pins make this microcontroller particularly suitable for industrial control and medical systems [1], [16].

The LPC2148 microcontroller has many features, some of which are describe as follows:

\section{(a) ARM7 Microprocessor}

The LPC2148 is embedded with ARM7TDMI-S microprocessor. The TDMI-S stands for 16-bit Thumb + JTAG Debug + fast Multiplier + enhanced ICE + Synthesizable core. Where T: supports both ARM (32-bit) and Thumb (16-bit) instruction sets, D: Contains Joint Test Action Group (JTAG) Debug extensions, M: Enhanced 32x8 Multiplier block, I: Embedded In-Circuit Emulator (ICE) macro cell, S: Synthesizable (i.e. distributed as Register Transfer Level (RTL) rather than a hardened layout). The ARM7TDMI-S is a general purpose 32-bit microprocessor, which offers high performance and very low power consumption.

The ARM architecture is based on Reduced Instruction Set Computer (RISC) principles, and the instruction set and related decode mechanism are much simpler than those of micro programmed Complex Instruction Set Computers (CISC). This simplicity results in a high instruction throughput and impressive real-time interrupt response from a small and cost-effective processor core. Pipeline techniques are employed so that all parts of the processing and memory systems can operate continuously. Typically, while one instruction is being executed, its successor is being decoded, and a third instruction is being fetched from memory.

The ARM7TDMI-S processor also employs a unique architectural strategy known as THUMB, which makes it ideally suited to high-volume applications with memory restrictions, or applications where code density is an issue.
The key idea behind THUMB is that of a super-reduced instruction set.

Essentially, the ARM7TDMI-S processor has two instruction sets:

- The standard 32-bit ARM instruction set.

- A 16-bit THUMB instruction set.

The THUMB set's 16-bit instruction length allows it to approach twice the density of standard ARM code while retaining most of the ARM's performance advantage over a traditional 16-bit processor using 16-bit registers. This is possible because THUMB code operates on the same 32-bit register set as ARM code. THUMB code is able to provide up to $65 \%$ of the code size of ARM, and $160 \%$ of the performance of an equivalent ARM processor connected to a 16-bit memory system [1], [16].

\section{(b) 10-Bit ADC}

The LPC2148 contain two analog to digital converters. These converters are single 10-bit successive approximation analog to digital converters. While ADC0 has six channels, ADC1 has eight channels. Therefore, total number of available ADC inputs for LPC2148 is 14 . Basic clocking for the A/D converters is provided by the APB clock. A programmable divider is included in each converter, to scale this clock to the $4.5 \mathrm{MHz}$ (max) clock needed by the successive approximation process. A fully accurate conversion requires 11 of these clocks [1], [16].

Features of ADC

- 10 bit successive approximation analog to digital converter (i.e. two in LPC2148).

- Input multiplexing among 6 or 8 pins (ADC0 and ADC1).

- Power-down mode.

- Measurement ranges $0 \mathrm{~V}$ to $\mathrm{V}_{\mathrm{REF}}$ (typically $3 \mathrm{~V}$; not to exceed $\mathrm{V}_{\mathrm{DDA}}$ voltage level).

- 10 bit conversion time $\geq 2.44 \mu \mathrm{s}$.

- Burst conversion mode for single or multiple inputs.

- Optional conversion on transition on input pin or Timer Match signal.

- Global Start command for both converters.

\subsubsection{Temperature Sensor LM35}

The LM35 series devices are precision integrated-circuit temperature sensors, with an output voltage linearly proportional to the Centigrade temperature. The LM35 device has an advantage over linear temperature sensors calibrated in Kelvin, as the user is not required to subtract a large constant voltage from the output to obtain convenient Centigrade scaling. The LM35 device does not require any external calibration or trimming to provide typical accuracies of $\pm 1 / 4{ }^{\circ} \mathrm{C}$ at room temperature and $\pm 3 / 4{ }^{\circ} \mathrm{C}$ over a full $-55^{\circ} \mathrm{C}$ to $150^{\circ} \mathrm{C}$ temperature range. Lower cost is assured by trimming and calibration at the wafer level. The low output impedance, linear output, and precise inherent calibration of the LM35 device makes interfacing to readout or control circuitry especially easy. The device is used with single power supplies, or with plus and minus supplies. As the LM35 device draws only $60 \mu \mathrm{A}$ from the supply, it has very low self-heating of less than $0.1^{\circ} \mathrm{C}$ in still air. The LM35 


\section{International Journal of Science and Research (IJSR) \\ ISSN (Online): 2319-7064}

Index Copernicus Value (2013): 6.14 | Impact Factor (2015): 6.391

device is rated to operate over a $-55^{\circ} \mathrm{C}$ to $150^{\circ} \mathrm{C}$ temperature range, while the $\mathrm{LM} 35 \mathrm{C}$ device is rated for a $-40^{\circ} \mathrm{C}$ to $110^{\circ} \mathrm{C}$ range [1], [17].

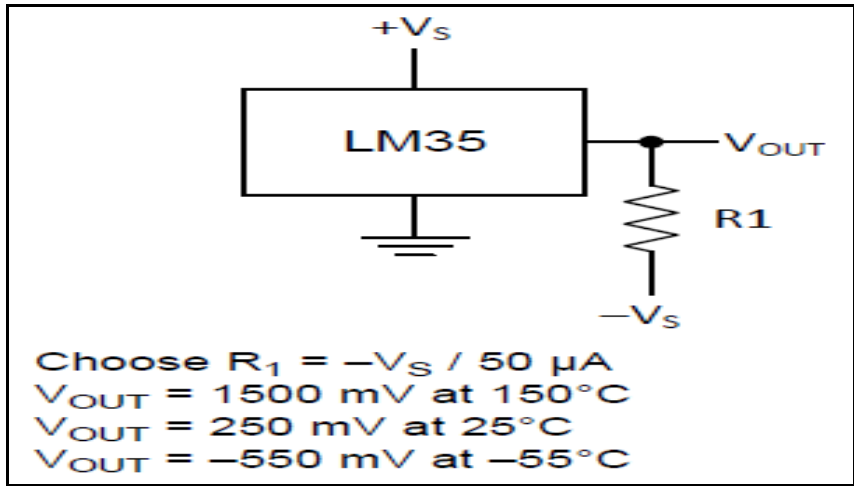

Figure 10: Full-Range Centigrade Temperature Sensor LM35

\subsubsection{Heartbeat Sensor IC LM358}

Heartbeat sensor is designed to give digital output of heartbeat when a finger is placed on it. This digital output is interfaced to LPC2148 microcontroller directly to measure the heartbeats in Beats per Minute (BPM) rate. It works on the principle of light modulation by blood flow through finger at each pulse. The Op-Amp IC LM358 is used in this Heartbeat Sensor prototype. The LM358 consists of two independent, high gain, internally frequency compensated operational amplifiers which were designed specifically to operate from a single power supply over a wide range of voltages [1], [5], [10], [11], [12]. The low-power, dual operational amplifiers are connected to a light detector/heartbeat sensing circuit consists of an IR LED (as Infrared Light Emitter/Transmitter) and an IR Photodiode (as Infrared Light Detector/Receiver). Out of two Op-Amps, one act as an amplifier and another used as comparator for the output of light detector/heartbeat sensing circuit. The IR LED used, is super bright, as the maximum light must pass spread in finger and detected by IR Photodiode. Now, when the heart pumps a pulse of blood through the blood vessels, the finger becomes slightly more opaque and so less light reached to the detector. With each heart pulse, the detector signal varies. This variation is converted to electrical pulse by IR Photodiode. And this electrical pulse is then amplified and triggered through Op-Amp IC LM358 which outputs $+5 \mathrm{~V}$ TTL/CMOS level signal or digital signal. The output signal is also indicated by a LED, which blinks on each heartbeat.

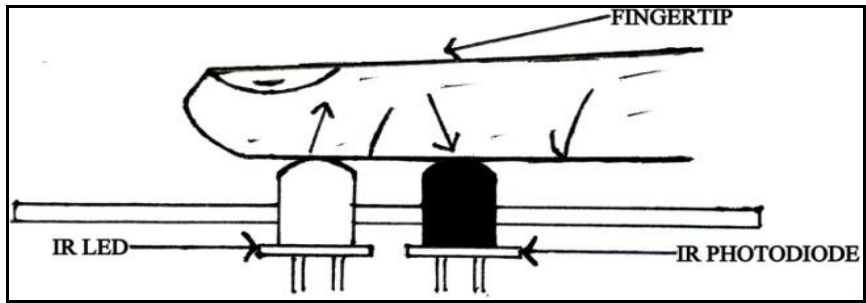

Figure 11: Heart Beat Measurement Principle

\subsubsection{GPS Module SIM28ML}

The concept of GPS is based on time and known position of specialized satellites. Far from North Pole and South Pole, a GPS unit can receive signals from 6 to 12 visible satellites at once. The satellites contain very stable atomic clocks that are synchronized to ground clocks and to each other also. The satellite locations are monitored precisely and any drift from true time, which is maintained on the ground is corrected daily. The GPS satellites continuously transmit their current time and position. A GPS receiver monitors multiple satellites and solves equations using trigonometry to determine the exact position of the receiver and its deviation from true time. There has to be at least four satellites in view for the GPS receiver to solve the geometric equations. A GPS receiver can calculate its position many times in one second. A GPS receiver calculates its speed and direction by using its change in position and change in time. Generally, the messages received by GPS receiver are in NMEA (National Marine Electronics Association) message format and the most commonly used NMEA protocol is NMEA-0183 protocol.

Table 1: Format of NMEA Output Messages

\begin{tabular}{|c|l|c|}
\hline NMEA Message Prefix & \multicolumn{1}{|c|}{ Description } & Direction \\
\hline \$GPGGA & Time, position and fix type data. & Out \\
\hline \$GPGL & Latitude, longitude, UTC time of position fix and status. & Out \\
\hline \$GPGSA & GNSS DOP and Active satellites & Out \\
\hline \$GPGSV & GNSS Satellites in View. & Out \\
\hline \$GPMSS & $\begin{array}{l}\text { Signal-to-noise ratio, signal strength, frequency, and bit rate } \\
\text { from a radio-beacon receiver. }\end{array}$ & Out \\
\hline \$GPRMC & Time, date, position, course and speed data. & Out \\
\hline \$GPVTG & Course and speed information relative to the ground. & Out \\
\hline \$GPZDA & $\begin{array}{l}\text { Precise Positioning Service (PPS) timing message } \\
\text { (synchronized toPPS). }\end{array}$ & Out \\
\hline
\end{tabular}

In this project, we used SIM28ML GPS receiver designed by SIMCom, which is use to track the location at anytime from anywhere. SIM28ML is a stand-alone or A-GPS (Assisted Global Positioning System) receiver with built-in low noise amplifier (LNA). Therefore, SIM28ML can relax antenna requirement and don't need for any external LNA. The SIM28ML can track as low as $-165 \mathrm{dBm}$ signal even without network assistance. The SIM28ML has excellent low power consumption characteristics i.e. for acquisition $16 \mathrm{~mA}$ and for tracking $15 \mathrm{~mA}$. SIM28ML supports various location and navigation applications, including autonomous GPS, QZSS, SBAS ranging (EGNOS, GAGAN, MSAS, and WAAS), RTCM, DGPS and A-GPS [1], [18].

Key features of SIM28ML:

- 22 tracking and 66 acquisition channels, up to 210 PRN channels.

- Small footprint: $10.1 * 9.7 * 2.5 \mathrm{~mm}, 18$-pin LCC package.

- 12 multi-tone active interference cancellers and jamming elimination.

- Indoor and outdoor multi-path detection and compensation.

- Max NMEA update rate up to $5 \mathrm{~Hz}$.

- Advanced software features:

1) EASY self-generated orbit prediction.

2) EPO/HotStill orbit prediction.

3) Always locate advanced location awareness technology.

\section{Volume 5 Issue 5, May 2016}




\section{International Journal of Science and Research (IJSR) \\ ISSN (Online): 2319-7064}

Index Copernicus Value (2013): 6.14 | Impact Factor (2015): 6.391

4) Supports logger function.

5) Supports Active Interference Cancellation (AIC).

- Pulse-per-second (PPS) GPS time reference:

1) Adjustable duty cycle.

2) Typical accuracy: $\pm 10 \mathrm{~ns}$.

- Interface: UART0/UART1.

- Operating temperature: $-40^{\circ} \mathrm{C} \sim+85^{\circ} \mathrm{C}$.

- Accuracy 2.5m CEP (Circular Error Probable).

- RoHS (Restriction of Hazardous Substances Directive) compliant.

The SIM28ML module provides complete signal processing from antenna input to host port in NMEA messages format. It requires $2.8 \mathrm{~V} \sim 4.3 \mathrm{~V}$ power supply. The host port is configurable to UART. The host data and I/O signal levels are $2.85 \mathrm{~V}$ CMOS compatible.

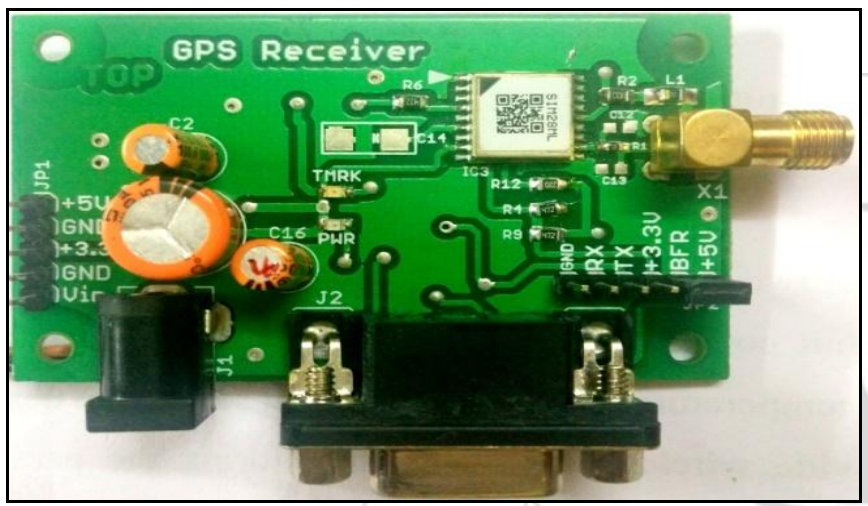

Figure 12: GPS Module SIM28ML

\subsubsection{ZigBee Module NORDIC nRF24L01+}

ZigBee is based on an IEEE 802.15.4 standard which is a packet-based radio protocol, use to create wireless personal area networks (WPANs). It is use to provide the communication which needs wireless applications that have low data rates and requires low power consumption. ZigBee has a defined rate of $250 \mathrm{Kbit} / \mathrm{s}$, which is best suited for intermittent data transmissions from a sensor or input device. This module has worldwide $2.4 \mathrm{GHz}$ ISM (The industrial, scientific and medical radio bands) bands and ultra low power operation. The ZigBee standard provides wireless networking, security, and application support services that operate on the basis of IEEE 802.15.4 Medium Access Control (MAC) and Physical Layer (PHY) wireless standard. It uses a suite of technologies which enable the selforganizing, self-healing and scalable networks that is efficient enough to manage various data traffic patterns. ZigBee is a low-cost, low-power, and wireless mesh networking standard device. The low cost allows this technology to be widely accepted in wireless control and monitoring applications. The low power consumption provides longer life with smaller batteries and the mesh networking provides high reliability and larger range of data transmission. ZigBee technology has been developed to fulfill the need of advanced wireless networking between various low power devices, sensors and modules [1], [13], [14], [15].

In our system, ZigBee module nRF24L01+ of Nordic Semiconductors is used. The nRF24L01+ is a single chip
2.4GHz transceiver with an embedded baseband protocol engine (Enhanced ShockBust ${ }^{\mathrm{TM}}$ ), suitable for ultra low power wireless applications. The nRF24L01+ is designed for operation in the world wide ISM frequency band at 2.400$2.4835 \mathrm{GHz}$. To design radio system with the nRF24L01+, we simply need an MCU and a few external passive components. We can operate and configure the nRF24L01+ through a Serial Peripheral Interface (SPI).The register map, which is accessible through SPI, contains all configuration registers in the nRF24L01+ and is accessible in all operation modes of the chip.

The embedded baseband protocol engine (Enhanced ShockBust ${ }^{\mathrm{TM}}$ ) is based on packet communication and support various modes from manual operation to advanced autonomous protocol operation. Internal FIFOs ensure a smooth data flow between the radio front end and the system's MCU. Enhanced ShockBust ${ }^{\mathrm{TM}}$ reduces system cost by handling all the high speed link layer operations.

The radio front end uses GSFK modulation. It has over configurable parameters like frequency channel, output power and air data rate. The nRF24L01+ supports an air data rate of $250 \mathrm{kbps}, 1 \mathrm{Mbps}$ and $2 \mathrm{Mbps}$. The high air data rate combined with two powers saving modes make the nRF24L01+ very suitable for ultra low power designs. The nRF24L01+ is drop in compatible with nRF24L01 and on air compatible with nRF2401A, nRF2402, nRF24E1 and nRF24E2. Intermodulation and wideband blocking values in nRF24L01+ are much improved in comparison to the nRF24L01 and the addition of the internal filtering to nRF24L01+ has improved the margins for meeting RF regulatory standards. Internal voltage regulators ensure a high Power Supply Rejection Ratio (PSRR) and a wide power supply [1], [19].

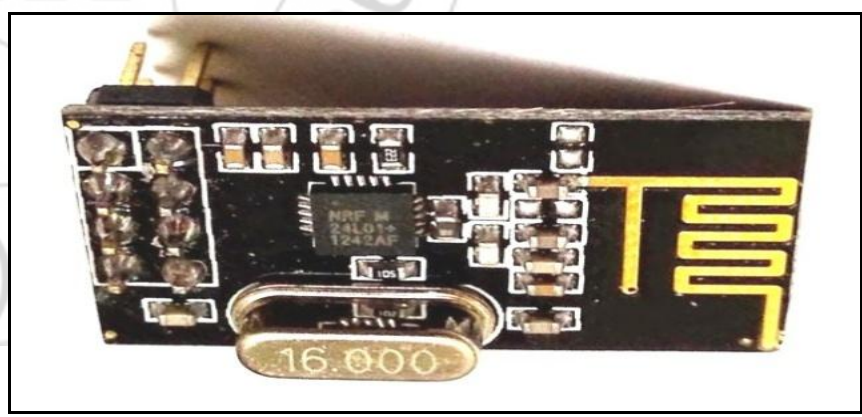

Figure 13: NORDIC nRF24L01+ Plug-in

\subsubsection{Panic Switch}

A panic switch is provided in this system, so that a soldier can request for his help from army control room and even from another fellow soldiers in panic situation by pressing it [1]. 


\section{International Journal of Science and Research (IJSR) \\ ISSN (Online): 2319-7064}

Index Copernicus Value (2013): 6.14 | Impact Factor (2015): 6.391

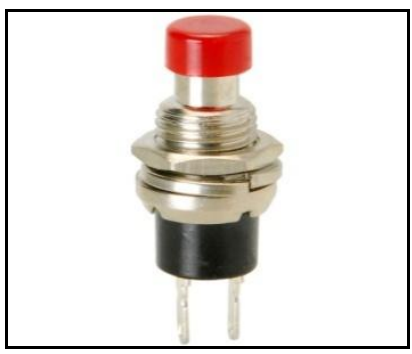

Figure 14: Panic Switch

\subsection{Software Description}

Software is a basic building block for the every system which designs the processing and operations. Following softwares are used in designing of this wireless embedded system.

- It includes PCB layout for our wireless monitoring system.

1) For PCB Layout: CadSoft EAGLE.

- It also includes the coding for LPC2148 and AT89S52 microcontroller.

1)For $\mu \mathrm{C}$ LPC2148: Embedded $\mathrm{C}$ language using Keil $\mu$ Vision 4 .

2)For $\mu \mathrm{C}$ AT89S52: Embedded $\mathrm{C}$ language using Keil $\mu$ Vision 3 .

- For Burning, the written programs into $\mu \mathrm{Cs}$, following softwares are used.

1) For $\mu$ C LPC2148: Flash Magic using PL-2303 Usb-toSerial CH340 Driver.

2) For $\mu \mathrm{C}$ AT89S52: Program ISP using USBasp LibUSB Win 32 Devices Driver.

- To display the data transmitted by soldiers unit on base station's PC through Control Room's Unit, we have used the following software.

1) For ZigBee with USB: AccessPort using PL-2303 Usbto-Serial CH340 Driver.

- We have also used coding for displaying the received data, through GUI (Graphical User Interface) on base station's PC.

1) For GUI: C\#.NET.

To design the circuit diagram, we used CadSoft EAGLE (Easily Applicable Graphical Layout Editor) software which is less complex, easy to learn, easy to understand and user friendly. It is the advance, simple and latest software for schematic electrical circuit designing and PCB layout. This is an expandable, flexible and scriptable EDA (Electronic Design Automation)/ ECAD (Electronic Computer Aided design) application with PCB layout editor, schematic capture editor, auto-router and CAM (Computer Aided Manufacturing) and BOM (Bill of Material) tools, developed by CadSoft Computer.

To write program using Embedded $\mathrm{C}$ programming language for ARM7 microprocessor, to process the functions of the peripherals like temperature sensor, heartbeat sensor, GPS and ZigBee modules, etc. we used Keil $\mu$ Vision software, which is an Integrated Development Environment (IDE) for ARM microprocessors. The Keil $\mu$ Vision IDE combines project management, run-time environment, program debugging, source code editing and build facilities in a single powerful environment. Keil $\mu$ Vision is easy-to-use and accelerates our embedded software development. Keil $\mu$ Vision supports multiple screens and allows us to create individual window layouts anywhere on the visual surface. The Keil $\mu$ Vision Debugger provides a single environment in which we can test, verify, and optimize our application code. The Debugger includes conventional features like simple and complex breakpoints, execution control, watch windows and provides full visibility to device peripherals.

The Graphical User Interface (GUI) is a type of interface which allows users to interact with the electronic devices through graphical icons and visual indicators. It displays the data, like location information, heartbeats and body temperature of each soldier, coming from control room's unit. For coding of GUI, we used C\#.NET (C Sharp.NET) programming language. The $\mathrm{C}$ Sharp, derived from $\mathrm{C}$ programming language, is a multi-paradigm programming language including strong typing, imperative, declarative, reflective, concurrent, functional, generic, structured, objectoriented (class-based), component-oriented, event-driven and task-driven programming disciplines. It introduces some unique and powerful features like delegates, lambda expressions, garbage collection, etc. It was developed by Microsoft within its .NET initiative and later approved as a standard by European Computer Manufacturers Association (ECMA-334) and International Organization for Standardization/ International Electrotechnical Commission (ISO/IEC 23270:2006). The C\# is a programming language designed for the Common Language Infrastructure such as .NET Framework, Portable.NET, free and open source-

Mono.

\section{Algorithm}

In mathematics, computer, electrical and electronic science, an Algorithm is a self-contained step-by-step set of operations, to be performed. The Algorithm of our wireless monitoring system is presented below:- 


\section{International Journal of Science and Research (IJSR) \\ ISSN (Online): 2319-7064}

Index Copernicus Value (2013): 6.14 | Impact Factor (2015): 6.391

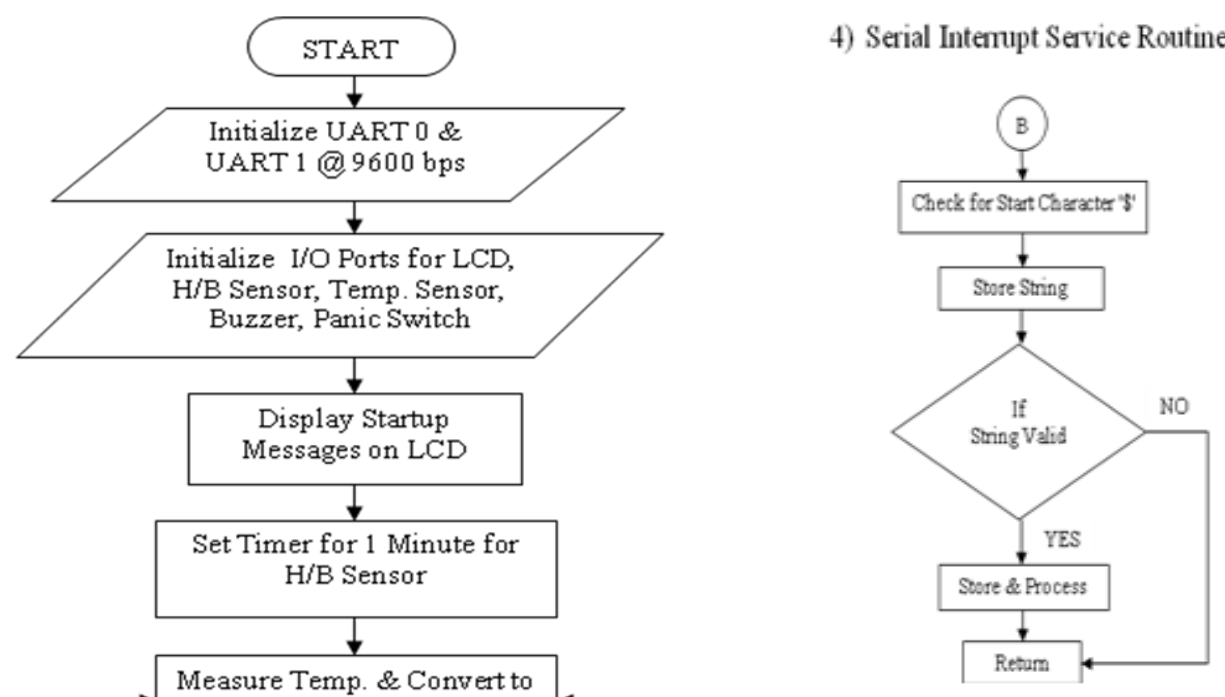

5) Hardware Extemal Intemupt
Service Routine (Panic Switch)

Figure 15: Flow Chart of the Wireless Health Monitoring

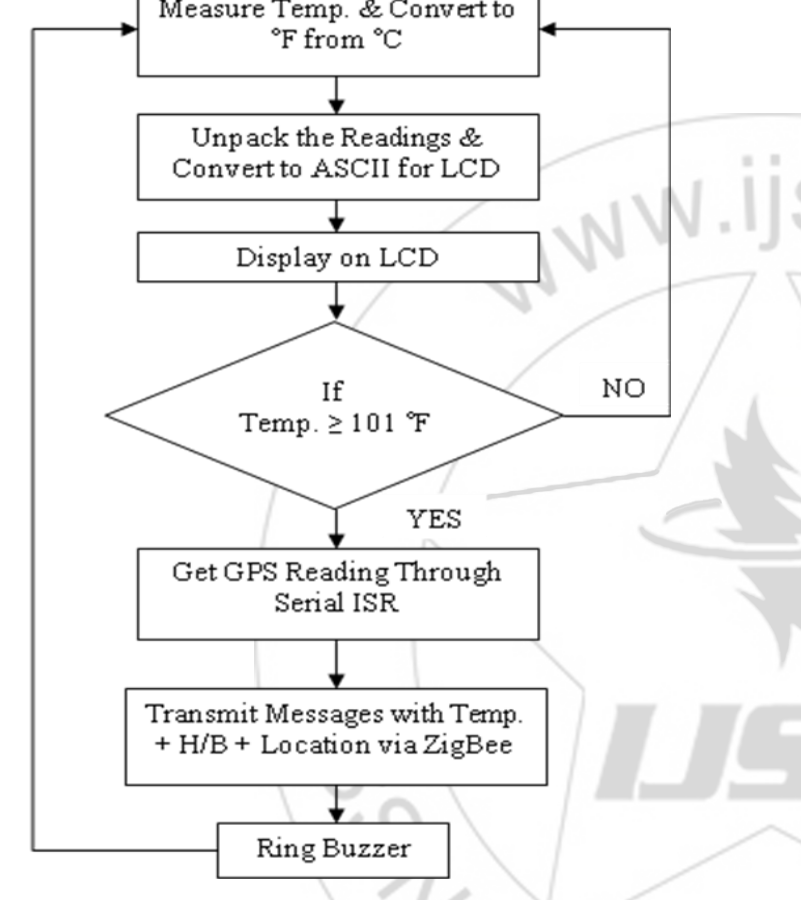

1) Hardware External Intemupt Service Routine

3) Process Routine

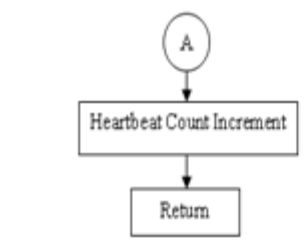

2) Timer/Intermupt Service Routine

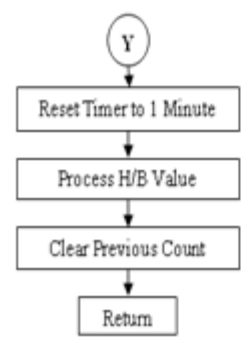

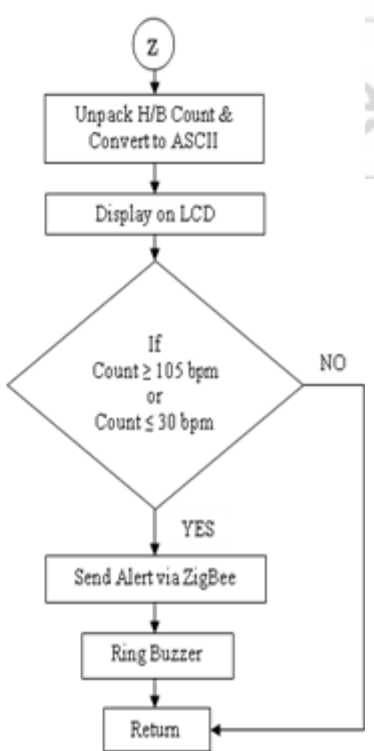

System

\section{Methodology}

In this project, our aim is to build the communication between soldiers and army control room and even with other fellow soldiers using wireless ZigBee Mesh Technology. Also to track the location of soldiers in the battlefield, in military search and rescue operations, and to provide real time health monitoring to soldiers. Therefore, in order to implement this project, we have used the GPS receiver to track the location of the soldiers through satellite, temperature sensor to sense the body temperature of the soldiers and heartbeat sensor to sense the heartbeat of the solders. We have used LPC2148 microcontroller which is embedded with ARM7 microprocessor to process all the received data, of Sensors and GPS receiver in real time. Also, ZigBee transceivers are used for wireless transmitting and receiving the data.

The temperature sensor LM35's output is directly interfaced with 10-bit ADC which is inbuilt in LPC2148 microcontroller. It will sense the body temperature of soldier and give that sensed analog signal to ADC, which convert that signal to digital signal. This digital signal is then process by ARM7 microprocessor. The processor converts the ${ }^{\circ} \mathrm{C}$ temperature to ${ }^{\circ} \mathrm{F}$, by the mathematical calculation written in its program. This output is then converted into ASCII to display the temperature on LCD provided.

The heartbeat sensor consists of two parts viz. light detector/heartbeat sensing circuit (i.e. IR LED and IR Photodiode) and IC LM358. The IR Photodiode/ IR Detector/IR Receiver's output is given to the Op-Amp 1 of IC LM358, which acts as a Negative Feedback Amplifier to amplify the signal level. A Pot 1 is connected to Op-Amp 1 to increase the gain. The output of Op-Amp 1 is given to the Op-Amp 2 of IC LM358, which acts as a Comparator. It compares the amplified output of Stage 1 with reference voltage set by Pot 2, to generate digital output. This digital output is then fed to microcontroller for processing, to count beats in per minute. This output is also converted into ASCII to display the heartbeats on LCD. 


\section{International Journal of Science and Research (IJSR) \\ ISSN (Online): 2319-7064 \\ Index Copernicus Value (2013): 6.14 | Impact Factor (2015): 6.391}

The processed digital output of the sensors are then compared with the predefined normal condition signals, and if any discrepancy occurs between sensed signals and defined normal signals, then it will be considered as an emergency.

The data of SIMCom SIM28ML device is initially TTL/CMOS voltage level, which gets converted into RS-232 voltage level data through IC MAX232 on board. Therefore, the data of GPS Receiver is RS-232 voltage level, which again gets converted into 5V TTL/CMOS voltage level data through IC MAX232, provided in soldier's Unit. This data is then passed to microcontroller to process.

When emergency occurs, ZigBee module takes data of heartbeat sensor, temperature sensor and GPS Receiver from microcontroller and sends to the army control room through ZigBee transceivers connected to each other wirelessly using ZigBee Mesh Topology. The ZigBee module also sends the help request to other fellow soldiers present in the ZigBee wireless range.

In any panic situation, to get help from control room and from other soldiers, when a soldier will press the panic switch, a message will send through ZigBee transceiver to army control room and to the other fellow soldiers present within the wireless range. As soon as another fellow soldier will receive a message, the buzzer provided in his system unit will generate sound.

The army control room's unit consists of a ZigBee transceiver with USB interface which is connected to the control room's PC. It will receive the data coming from soldiers ZigBee modules and give it to the PC through USB. The PC will display the received data on the screen through Windows based software called as GUI (Graphical User Interface). The PC can also display the received data through AccessPort software.

\section{Results}

The results of our wireless monitoring system using ZigBee Mesh Technology are shown through AccessPort and GUI on computer screen.

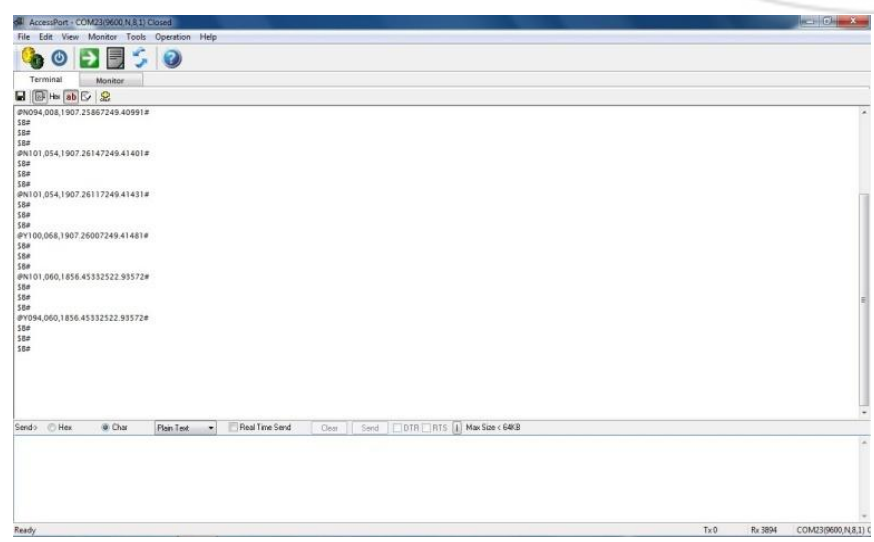

Figure 16: Result of both Soldier's Unit through AccessPort

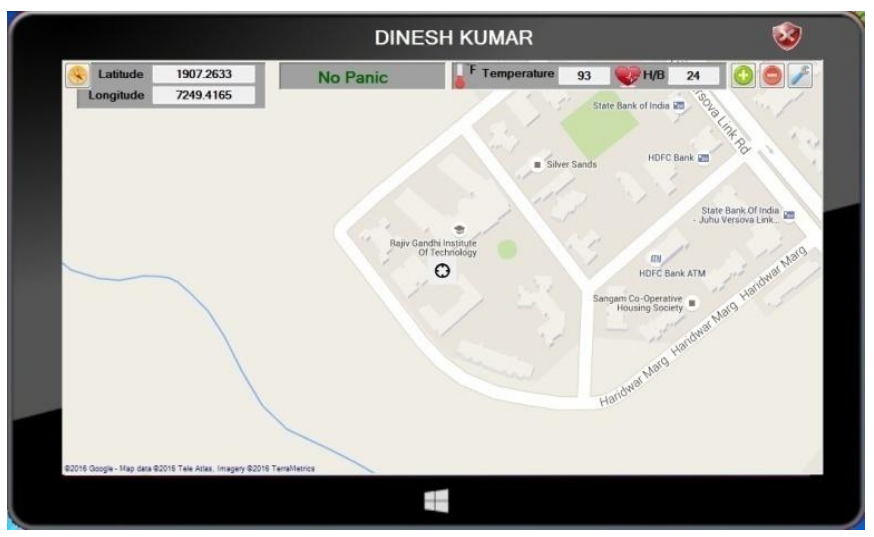

Figure 17: Result of Soldier's Unit 1 through GUI with Low Heartbeats

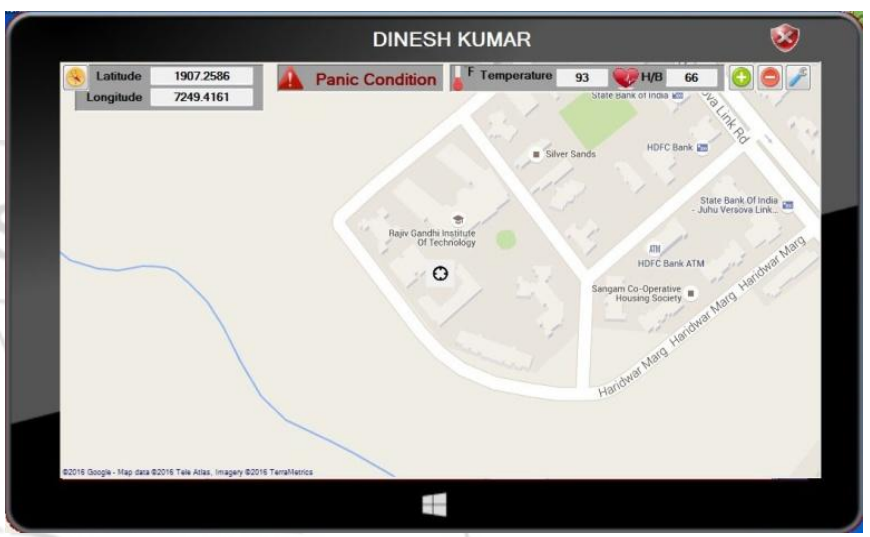

Figure 18: Result of Soldier's Unit 1 through GUI in Panic Condition

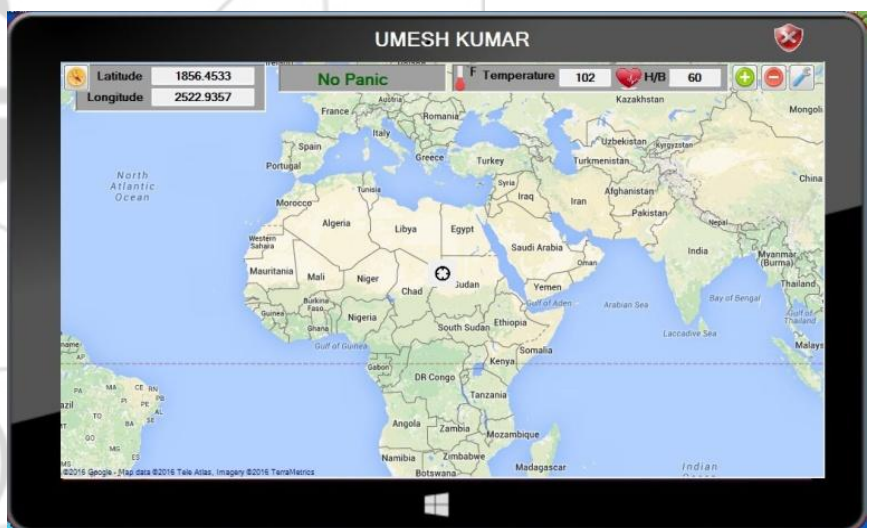

Figure 19: Result of Soldier's Unit 2 through GUI with High Body Temperature

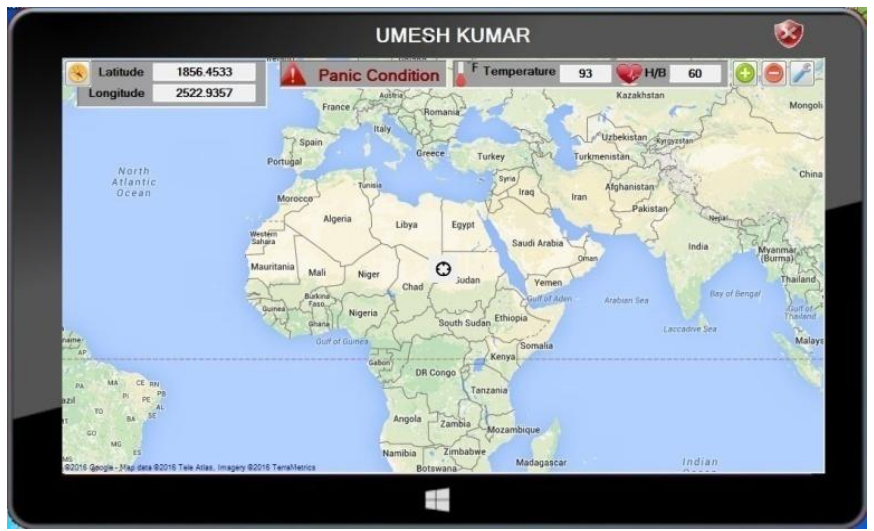

Figure 20: Result of Soldier's Unit 2 through GUI in Panic Condition

Volume 5 Issue 5, May 2016 www.ijsr.net 


\section{International Journal of Science and Research (IJSR) \\ ISSN (Online): 2319-7064}

Index Copernicus Value (2013): 6.14 | Impact Factor (2015): 6.391

\section{Conclusion}

From this wireless embedded system, we can conclude that we are able to transmit data which is sensed from remote soldier, to army control room using ZigBee Mesh Topology through ZigBee transceivers as a wireless transmission technology. This system provides health monitoring for soldiers using heartbeat sensor to sense heartbeats and temperature sensor to sense body temperature of soldiers. This system is completely integrated and can track the location of soldier at anytime from anywhere on the earth using GPS receiver. Also, it helps the soldier to get help from army base station and from another fellow soldier in panic situation using Panic Switch. This system provides the location information and health parameters of soldier in real time to the army control room. Due to use of wireless ZigBee technology, this system is very useful to military forces during wars, search and rescue operations, etc. as it can be used in warfield without any network restriction. Thus, this system provides high level security and safety to our soldiers.

\section{References}

[1] Dineshkumar Jaiswar, Sanjna S. Repal, "Real Time Tracking and Health Monitoring of Soldiers using ZigBee Technology: a Survey", International Journal of Innovative Research in Science, Engineering and Technology, ISSN: 2319-8753, Volume 4, Issue 7, July 2015, DOI: 10.15680/IJIRSET.2015.0407100, pp: (5560-5574).

[2] Hock Beng Lim, Di Ma, Bang Wang, Zbigniew Kalbarczyk, Ravishankar K. Iyer, Kenneth L. Watkin, "A Soldier Health Monitoring System for Military Applications", 2010 International Conference on Body Sensor Networks, 978-0-7695-4065-8/10/\$26.00 (C) 2010 IEEE, DOI: 10.1109/BSN.2010.58, pp: (246-249).

[3] William Walker, A. L. Praveen Aroul, Dinesh Bhatia, "Mobile Health Monitoring Systems", 31st Annual International Conference of the IEEE EMBS, Minneapolis, Minnesota, USA, September 2-6, 2009, 978-1-4244-3296-7/09/\$25.00 @ 2009 IEEE, pp: (51995202).

[4] M. Pranav Sailesh, C. Vimal Kumar, B. Cecil, B. M. Mangal Deep, P. Sivraj, "Smart Soldier Assistance using WSN", International Conference on Embedded Systems (ICES 2014), 978-1-4799-5026-3/14/\$31.00 (C) 2014 IEEE, pp: (244-249).

[5] P.S. Kurhe, S.S. Agrawal, "Real Time Tracking and Health Monitoring System of Remote Soldier Using ARM 7", International Journal of Engineering Trends and Technology, ISSN: 2231-5381, Volume 4, Issue 3, No. 1, March 2013, pp: (311-315).

[6] Shruti Nikam, Supriya Patil, Prajkta Powar, V.S. Bendre, "GPS Based Soldier Tracking and Health Indication System", International Journal of Advanced Research in Electrical, Electronics and Instrumentation Engineering, ISSN: 2278-8875, Volume 2, Issue 3, March 2013, pp: (1082-1088).

[7] Prof. Pravin Wararkar, Sawan Mahajan, Ashu Mahajan, Arijit Banerjee, Anchal Madankar, Ashish Sontakke, "Soldier Tracking and Health Monitoring System", The
International Journal of Computer Science \& Applications, ISSN: 2278-1080, Volume 2, No. 02, April 2013, pp: (81-86).

[8] Govindaraj A., Dr. S. Sindhuja Banu, "GPS Based Soldier Tracking and Health Indication System with Environmental Analysis", International Journal of Enhanced Research in Science Technology \& Engineering, ISSN: 2319-7463, Volume 2, Issue 12, December 2013, pp: (46-52).

[9] Palve Pramod, "GPS Based Advanced Soldier Tracking With Emergency Messages \& Communication System”, International Journal of Advance Research in Computer Science and Management Studies, ISSN: 2321-7782, Volume 2, Issue 6, June 2014, pp: (25-32).

[10] Mr. Rajdeep Limbu, Prof. V. V. Kale, "GPS Based Soldier Tracking and Health Monitoring System", International Journal for Technological Research in Engineering, ISSN: 2347-4718, Volume 1, Issue 12, August 2014, pp: (1485-1488).

[11] Rubina.A.Shaikh, "Real Time Health Monitoring System of Remote Patient Using Arm7", International Journal of Instrumentation, Control and Automation, ISSN: 22311890, Volume 1, Issue 3-4, April 2012, pp: (102-105).

[12] Ekta Madhyan, Mahesh Kadam, "A Unique Health Care Monitoring System Using Sensors and ZigBee Technology", International Journal of Advanced Research in Computer Science and Software Engineering, ISSN: 2277-128X, Volume 4, Issue 6, June 2014, pp: (501-509).

[13]Dr. S. S. Riaz Ahamed, "The Role of ZigBee Technology in Future Data Communication System", Journal of Theoretical and Applied Information Technology, ISSN: 1817-3195, Volume 5, No. 2, February 2009, pp: (129-135).

[14] Nisha Ashok Somani, Yask Patel, "ZigBee: A Low Power Wireless Technology for Industrial Applications", International Journal of Control Theory and Computer Modeling, ISSN: 2249-1155, Volume 2, No. 3, May 2012, DOI: 10.5121/ijctcm.2012.2303, pp: (27-33).

[15]P. Rohitha, P. Ranjeet Kumar, Prof. N. Adinarayana, Prof. T. Venkat Narayana Rao, "Wireless Networking through ZigBee Technology", International Journal of Advanced Research in Computer Science and Software Engineering, ISSN: 2277-128X, Volume 2, Issue 7, July 2012, pp: (49-54).

[16] NXP Semiconductors, UM10139, LPC 214X User Manual, Rev. 4, 23 April 2012, pp: (1-354).

[17] Texas Instruments Inc., LM 35 Datasheet, SNIS159EAugust 1999-Revised January 2015, pp: (1-31).

[18] SIMCom Wireless Solutions Ltd., SIM28ML Hardware Design V1.01, July 2014, pp: (1-28).

[19] Nordic Semiconductor, nRF24L01+ Single Chip 2.4GHz Transceiver, Product Specification V1.0, September 2008, pp: (1-78).

\section{Authors Profile}

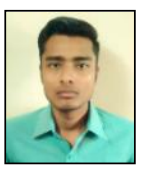

Dineshkumar L. Jaiswar is currently pursuing M.E. in Electronics and Telecommunication from MCT's Rajiv Gandhi Institute of Technology, Mumbai University. His area of interest is Wireless Communication and Embedded System design and 


\section{International Journal of Science and Research (IJSR) \\ ISSN (Online): 2319-7064 \\ Index Copernicus Value (2013): 6.14 | Impact Factor (2015): 6.391}

implementation. He has completed his B.E. in Electronics and Telecommunication from AET's Atharva College of Engineering, Mumbai University.

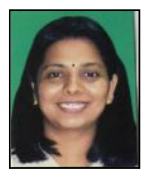

Sanjna S. Repal is currently working as Assistant Professor in MCT's Rajiv Gandhi Institute of Technology, Mumbai University. Her area of interest is design of Microprocessor, Microcontroller and VLSI circuits and systems. She has completed her B.E. in Electrical Engineering from Sardar Patel College of Engineering, Mumbai University and M.E. in Electronics Engineering from Fr. Conceicao Rodrigues College of Engineering, Mumbai University.

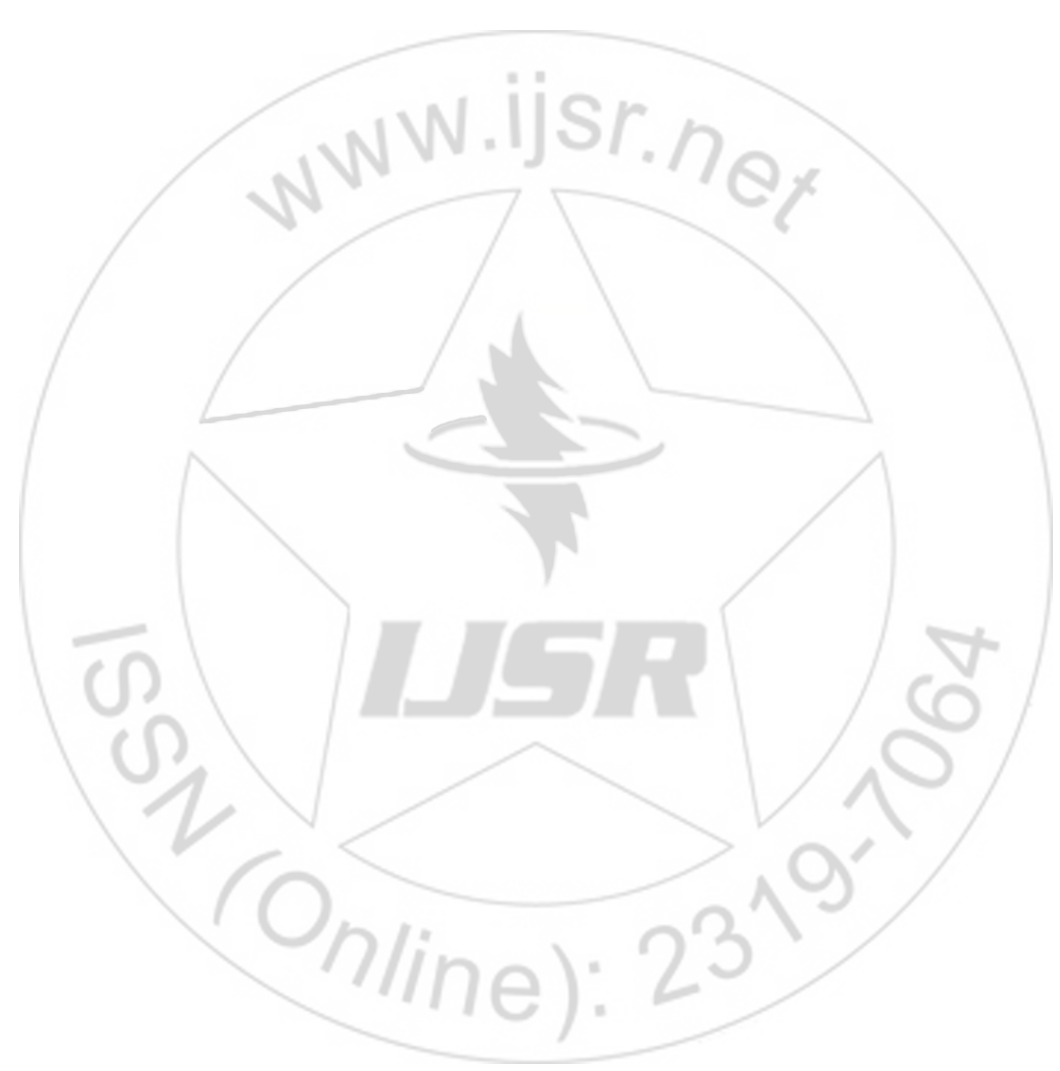

Volume 5 Issue 5, May 2016 www.ijsr.net 